\title{
Diabetes en Medicina Interna
}

Gregorio Marañón, clínico insigne y pionero de la Endocrinología española, afirmó que "quien quiera aprender Medicina, que estudie la diabetes". Con ello, quería resaltar el carácter multisistémico de la enfermedad, su heterogeneidad clínica y su gran complejidad fisiopatológica. Dicha aseveración sigue plenamente vigente en la actualidad. Lo que Marañón no pudo pronosticar fue el carácter epidémico que la diabetes ha adquirido en pocas décadas, debido a que la pandemia de obesidad y el envejecimiento de la población han incrementado de forma exponencial la prevalencia mundial de diabetes mellitus tipo 2, convirtiéndola en un problema de salud pública de gran magnitud. Actualmente, la prevalencia de diabetes en España es del 14\%' y se predice que aumentará un $22 \%$ en las próximas tres décadas, siendo la población mayor de 70 años la que sufrirá un mayor incremento². Se estima que cada 6 segundos se produce una muerte por causa de la diabetes, en su mayoría por enfermedad cardiovascular³. Según cálculos de la Organización Mundial de la Salud (OMS), la diabetes provocará en el futuro próximo mayores pérdidas de años de vida que el tabaquismo o el SIDA4. Actualmente, la diabetes es responsable del $12 \%$ de los costes sanitarios globales y su creciente impacto económico puede comprometer la sostenibilidad de los sistemas públicos de salud's.

La Revista Española de Casos Clínicos en Medicina Interna (RECCMI) nos ofrece un número monográfico con los mejores casos clínicos en diabetes seleccionados en un concurso nacional de casos clínicos promovido por la Sociedad Española de Medicina Interna (SEMI). En su gran mayoría, los autores son médicos residentes de nuestra especialidad que encuentran en este formato una forma atractiva y asequible de introducirse en la escritura científica. Estos casos abordan un amplio abanico de situaciones clínicas que incluyen tanto desafíos diagnósticos como complicaciones infrecuentes o los aspectos más prevalentes que le siguen, representan un reto asistencial.

La diabetes tipo 2 podría definirse como un síndrome pluripatológico. De hecho, se ha comunicado que casi el 90\% de los pacientes con diabetes tipo 2 en nuestro medio presentan multicomorbilidad ${ }^{6}$, lo que resalta la importancia de realizar un enfoque integral e individualizado de esta enfermedad.
Los últimos consensos de referencia sobre el tratamiento de la diabetes tipo $2^{7}$ han abandonado el enfoque glucocéntrico de la enfermedad, que prioriza como objetivo el grado de control de la HbAlc, y se centra en las características del paciente (existencia de enfermedad cardiovascular, insuficiencia cardíaca o insuficiencia renal; obesidad; riesgo de hipoglucemia), una perspectiva que resulta mucho más cercana para los médicos internistas.

La Medicina Interna juega un papel central en el abordaje de la diabetes en España. Según los últimos datos disponibles, correspondientes al año 2015 y facilitados por el Ministerio de Sanidad, los servicios de Medicina Interna son, con gran diferencia, los servicios médicos que atienden a más pacientes diabéticos hospitalizados en España. En concreto, los médicos internistas son los responsables del $34 \%$ de las altas hospitalarias de los pacientes diabéticos ingresados por cualquier causa. Tras la Medicina Interna, las especialidades que atienden a más pacientes hospitalizados con diabetes son Cardiología (9\% de las altas hospitalarias), Cirugía (9\%), Traumatología (7\%) y Urología (5\%). En conjunto, los servicios de Medicina Interna atienden a más pacientes hospitalizados que todos los demás servicios médicos (27\%) y que la globalidad de los servicios quirúrgicos $(27 \%)^{8}$. Además, como era esperable, los pacientes ingresados en Medicina Interna tienen una mayor edad media (77 años) y una mayor carga de comorbilidad que los hospitalizados en otras unidades ${ }^{8}$. Igualmente, el protagonismo de la Medicina Interna en el manejo de las complicaciones cardiovasculares de los pacientes con diabetes es indiscutible. Así, el $42 \%$ de los pacientes diabéticos hospitalizados por enfermedades cardiovasculares ingresan en servicios de Medicina Interna. El porcentaje de altas hospitalarias por insuficiencia cardíaca, ictus y cardiopatía isquémica a cargo de Medicina Interna es del 56\%, 41\% y $29 \%$, respectivamente ${ }^{8}$.

El carácter holístico y versátil de nuestra especialidad explica que los internistas hayamos sido siempre la fuerza de choque ante las principales alertas sanitarias. De los servicios de Medicina Interna surgió la respuesta a la epidemia de SIDA. Y, con toda seguridad, los servicios de Medicina Interna sabrán dar respuesta al actual desafío que representa la diabetes para nuestro sistema nacional de salud.

\section{Ricardo Gómez-Huelgas Servicio de Medicina Interna. Hospital Regional Universitario de Málaga. Málaga. España. Presidente de la Sociedad Española de Medicina Interna}

Citar como: Gómez-Huelgas R. Diabetes en Medicina Interna. Rev Esp Casos Clin Med Intern (RECCMI). 2019 (Ene); 4(Supl 1): 1-2. doi: 10.32818/reccmi.a4s1a1. 


\section{Bibliografía}

1. Soriguer F, Goday A, Bosch-Comas A, Bordiú E, Calle-Pascual A, Carmena R, et al. Prevalence of diabetes mellitus and impaired glucose regulation in Spain: the Di@bet.es Study. Diabetologia. 2012; 55: 88-93.

2. IDF Diabetes Atlas Eight Edition. International Diabetes Federation. 2017.

3. Roger VL, Go AS, Lloyd-Jones DM, Adams RJ, Berry JD, Brown TM, et al; American Heart Association Statistics Committee and Stroke Statistics Subcommittee. Heart disease and stroke statistics 2011 update: a report from the American Heart Association. Circulation. 2011; 123: e18-e209.

4. World Health Statistics 2015. World Health Organization.

5. Zhang P, Zhang X, Brown J, Vistisen D, Sicree R, Shaw J, et al. Global healthcare expenditure on diabetes for 2010 and 2030. Diabetes Res Clin Pract. 2010; 87: 293-301. Erratum in: Diabetes Res Clin Pract. 2011; 92: 301.
6. Alonso-Morán E, Orueta JF, Esteban Jl, Axpe JM, González ML, Polanco NT, et al. Multimorbidity in people with type 2 diabetes in the Basque Country (Spain): prevalence, comorbidity clusters and comparison with other chronic patients. Eur J Intern Med. 2015; 26: 197-202

7. Davies MJ, D'Alessio DA, Fradkin J, Kernan WN, Mathieu C, Mingrone G, et al. Management of hyperglycemia in type 2 diabetes, 2018. A consensus report by the American Diabetes Association (ADA) and the European Association for the Study of Diabetes (EASD). Diabetes Care. 2018; 41: 2669-2701.

8. Zapatero-Gaviria A, Gómez-Huelgas R, Canora-Lebrato J, Ena-Muñoz J, Romero-Sánchez M, Mendez-Bailón M, et al. Analysis of hospitalizations by cardiovascular disease in the population with diabetes in Spain. Rev Clin Esp. 2018 Nov 15. pii: S0014-2565(18)30281-9. 